\title{
Desfecho perinatal em gestantes usuárias de drogas atendidas em um centro especializado
}

\author{
Marcos Benatti Antunes ${ }^{1,2}$ \\ Marcela de Oliveira Demitto ${ }^{3}$ \\ Camila Padovani ${ }^{1,4}$ \\ Kelye Cristina de Moura Elias ${ }^{4}$ \\ Antonio Carlos Monteiro de Miranda ${ }^{1}$ \\ Sandra Marisa Pelloso ${ }^{1}$
}

Objetivo: analisar as repercussões perinatais do uso de drogas por gestantes atendidas em um ambulatório de alto risco. Método: estudo caso-controle, no qual foram avaliados 920 prontuários, no período de 2012-2013. O grupo caso foi constituído pelas gestantes usuárias de drogas lícitas/ilícitas (41) e o controle por gestantes de risco não usuárias (82). Resultados: as gestantes usuárias apresentaram risco aumentado para prematuridade $(R R=2,64, p=0,02)$, baixo peso ao nascer $(R R=5,42, p=0,01)$ e baixo índice de Apgar no $1^{\circ}$ minuto $(R R=2,97$, $p=0,01$ ). Conclusão: os resultados indicam que gestantes usuárias de drogas apresentam desfechos perinatais desfavoráveis à gestação.

Descritores: Enfermagem Materno-Infantil; Drogas de Abuso; Gravidez; Complicações na Gravidez.

\footnotetext{
${ }^{1}$ Universidade Estadual de Maringá, Centro de Ciências da Saúde, Maringá, PR, Brasil.

2 Prefeitura Municipal de Sarandi, Secretaria Municipal de Saúde, Sarandi, PR, Brasil.

${ }^{3}$ Centro Universitário Cesumar, Maringá, PR, Brasil.

${ }^{4}$ Santa Casa de Maringá, Maringá, PR, Brasil.
} 


\title{
Perinatal outcomes in pregnant drug users attended at a specialized center
}

Objective: to analyze the perinatal repercussions of drug use by pregnant women treated in a high risk outpatient clinic. Method: a case-control study in which 920 medical records were evaluated in the period 2012-2013. The case group consisted of pregnant women who used licit/illicit drugs (41) and the control group of non-user pregnant women (82). Results: pregnant women using drugs presented increased risk for prematurity $(R R=2.64, p=0.02)$, low birth weight $(R R=5.42, p=0.01)$ and low one-minute Apgar score $(R R=2.97, p=0.01)$. Conclusion: the results indicate that pregnant women who use drugs have unfavorable perinatal outcomes during gestation.

Descriptors: Maternal-Child Nursing; Street Drugs; Pregnancy; Pregnancy Complications.

\section{Los resultados perinatales en mujeres embarazadas usuarios de drogas en un centro especializado}

\begin{abstract}
Objetivo: analizar los resultados perinatales de consumo de drogas por mujeres embarazadas en una clínica de alto riesgo. Método: estudio de casos y controles, que evaluó 920 registros médicos, en 2012-2013. El grupo de casos estaba compuesto de las mujeres embarazadas que drogas lícitas/ilegales (41) y el control del riesgo de las mujeres embarazadas no consumidores (82). Resultados: las mujeres embarazadas que se encontraban en mayor riesgo de parto prematuro $(R R=2,64, p=0,02)$, bajo peso al nacer $(R R=5,42, p=0,01)$ y la puntuación de Apgar baja a 1 minuto $(R R=2,97, p=0,01)$. Conclusión: los resultados indican que las mujeres embarazadas que tienen las drogas adversos del embarazo los resultados perinatales.

Descriptores: Enfermaría Maternoinfantil; Drogas Ilícitas; Embarazo; Complicaciones del Embarazo.
\end{abstract}

\section{Introdução}

O consumo de drogas lícitas/ilícitas tem aumentado e repercutido em todo o mundo, tanto em homens quanto em mulheres, ocupando lugar entre as 20 principais causas de problemas de saúde catalogadas pela Organização Mundial de Saúde (OMS), sendo considerado um problema significativo de vulnerabilidade e de saúde pública, refletindo na sociedade por meio de mudanças no estado de saúde mental e social dos usuários ${ }^{(1)}$. Embora os homens apresentem maior tendência (três vezes a mais) no uso de drogas comparados com as mulheres, tem sido frequente a identificação de gestantes usuárias de drogas lícitas/ilícitas pelos profissionais de saúde, o que torna o problema mais acentuado, uma vez que a exposição na gravidez pode comprometer a integridade não só da mãe, mas também do feto ${ }^{(2-3)}$.

Estima-se que um pouco mais de $5 \%$ da população mundial (246 milhões de pessoas) na faixa etária de 15 a 64 anos tenha feito uso de drogas em 2013, em uma proporção de uma mulher para cada três usuários homens, totalizando 187.100 mortes em ambos os sexos neste mesmo ano(2), entretanto não existem dados estatísticos mundiais de prevalência de gestantes usuárias de drogas e dos desfechos perinatais.

No período gestacional, o uso de drogas lícitas/ ilícitas é considerado uma condição clínica preexistente, sendo, portanto, um risco para o binômio (mãe/feto). Em um levantamento epidemiológico descrito no Manual 
de Técnico de Gestação de Alto Risco do Ministério da Saúde (MS), em 108 cidades e com mais de 200.000 habitantes, a prevalência de dependentes de álcool foi de $12,3 \%$, sendo $6,9 \%$ em mulheres, destas $12,1 \%$ na faixa de 18 a 24 anos e $7,7 \%$ de 25 a 34 anos, ou seja, o uso de álcool aumenta no período de fertilidade(4).

Em estudo realizado no Paraná com 394 mulheres, $18,28 \%$ relataram fazer uso de drogas durante a gestação, apontando o cigarro e o álcool como as mais utilizadas $^{(3)}$. Já em outro estudo realizado no Acre com 1797 consultas de pré-natal, foram identificadas 100 $(5,56 \%)$ gestantes que faziam uso de drogas, entre elas $2,61 \%$ faziam uso de crack, $2,05 \%$ consumiam bebida alcoólica, 1,22\% fumava maconha, $1,00 \%$ era fumante de cigarro e $0,94 \%$ fazia uso de cocaína ${ }^{(5)}$.

As gestantes expostas a essas substâncias apresentam maiores incidências de complicações clínicas e obstétricas, realizando menor número de consulta de pré-natal e maior número de hospitalizações ${ }^{(6)}$. Além disso, existe o risco de descolamento prematuro de placenta, ansiedade, distúrbios afetivos e em alguns casos até aborto $^{(7)}$.

Diante desse cenário e da problematização aqui levantada da relação gestação e uso de drogas lícitas/ilícitas, o objetivo deste estudo foi analisar as repercussões perinatais do uso de drogas por gestantes atendidas em um ambulatório de alto risco.

\section{Método}

Estudo exploratório, retrospectivo, de abordagem quantitativa do tipo caso-controle, que teve como fonte de dados prontuários e carteirinhas de 920 gestantes classificadas como alto risco e atendidas no período de 2012-2013, em um ambulatório do Sul do Brasil. Os dados referentes aos resultados perinatais foram obtidos por meio do livro de registro de partos da maternidade de referência vinculada a esse ambulatório.

Os critérios de inclusão foram: parto com idade gestacional superior a 20 semanas; gestantes classificadas como alto risco pela Atenção Primária de Saúde (APS); e ter realizado o parto no hospital de referência vinculado a esse ambulatório.

As gestantes foram divididas em dois grupos, Grupo Caso e Grupo Controle. O primeiro foi constituído por todas as gestantes incluídas no ambulatório de risco com condição preexistente de uso de drogas lícitas/ ilícitas e que deu à luz na referida maternidade. No Grupo Controle, incluíram-se as gestantes que foram atendidas no ambulatório de risco com qualquer risco, exceto pela condição preexistente de uso de drogas lícitas/ilícitas, que deram à luz na mesma maternidade, atendidas subsequentes e homogeneizadas de acordo com a idade das gestantes do Grupo Caso, além disso, a amostra foi emparelhada um caso para dois controles com o intuito de garantir uma maior comparabilidade entre os grupos.

Para a coleta de dados, utilizou-se um formulário predeterminado, construído com base no Manual de Gestação de Alto Risco preconizado pelo $\mathrm{MS}^{(4)}$, bem como de conhecimentos prévios relacionados às informações contidas nos prontuários do serviço. Após a coleta dos dados, estes foram transcritos em uma planilha do programa Microsoft Office Excel $2010^{\circledR}$.

As análises foram realizadas entre o Grupo Caso (GCA): gestantes usuárias de drogas lícitas/ilícitas e o Grupo Controle (GCO): não usuárias de drogas lícitas/ ilícitas. As variáveis do estudo foram: Baixo peso ao nascer (BPN) (Recém-nascido com peso < $2.500 \mathrm{~g}$ ); Apgar $1^{\circ}$ minuto (menores que 7 ); Apgar $5^{\circ}$ minuto (menores que 7); Prematuridade (nascidos antes de 37 semanas); Parto Normal e Parto Cesárea.

A análise estatística foi realizada com auxílio do Software Epi Info versão 3.5.1, comparando-se o risco relativo (RR) no intervalo de confiança (IC) a 95\%, e para associação das variáveis, utilizou-se o teste Quiquadrado e Exato de Fisher ao nível de significância quando $\mathrm{p}<0,05$.

O estudo respeitou as Diretrizes e Normas Regulamentadoras de Pesquisas Envolvendo Seres Humanos do Conselho Nacional de Saúde (resolução CNS 466/2012) e foi aprovado conforme parecer $n^{\circ} 681317 / 2014$.

\section{Resultados}

Entre os 920 prontuários de gestantes que participaram do estudo, 5,8\% (53) apresentaram como condição preexistente o uso de drogas lícitas/ilícitas, o que fez com que elas fossem encaminhadas para o ambulatório de alto risco para o acompanhamento do pré-natal especializado. Dessas gestantes, 4,5\% (41) tiveram o parto no hospital de referência e foram incluídas no estudo. O GCO foi selecionado a partir das 920 gestantes que não apresentaram como risco o uso de drogas, pareando por idade duas por uma do GCA, resultando em 82 (8,9\%), conforme Figura 1.

Em ambos os grupos, a faixa etária predominante foi de 19 a 35 anos (68,3\%). Com relação à raça/ cor branca, constatou-se $58,5 \%$ no GCA e $68,3 \%$ no GCO. No que tange à situação conjugal, o GCA teve a predominância de mulheres que não vivem com um parceiro $(61,0 \%)$, enquanto que no GCO houve uma prevalência de mulheres que vivem com 
o companheiro $(62,2 \%)$. No aspecto escolaridade, em ambos os grupos, as gestantes cursaram menos que 8 anos, tendo o GCA $56,1 \%$ e o GCO $57,3 \%$. Os resultados referentes à renda familiar apontaram que a maioria das mulheres usuárias de drogas lícitas/ilícitas (56,1\%) não possuía nenhum rendimento; já no GCO, a maioria $(53,7 \%)$ tinha como renda de um a dois salários mínimos. Em ambos os grupos, as gestantes realizaram de uma a três consultas no ambulatório especializado, GCA 56,0\% e no GCO 47,6\%. Em relação ao número de gestações, incluindo a atual, constatou-se que no
GCA teve a predominância de quatro a cinco gestações $(36,6 \%)$ e no GCO de duas a três gestações $(48,8 \%)$ (Tabela 1).

Ao analisar os grupos, as mulheres que possuíam como condição clínica preexistente o uso de drogas lícitas/ilícitas apresentaram risco aumentado para prematuridade $(R R=2,64, p=0,02)$, baixo peso ao nascer $(R R=5,42, p=0,01)$ e baixo Índice de Apgar no $1^{\circ}$ minuto $(R R=2,97, p=0,01)$ quando comparadas com as gestantes de alto risco do grupo controle (Tabela 2).

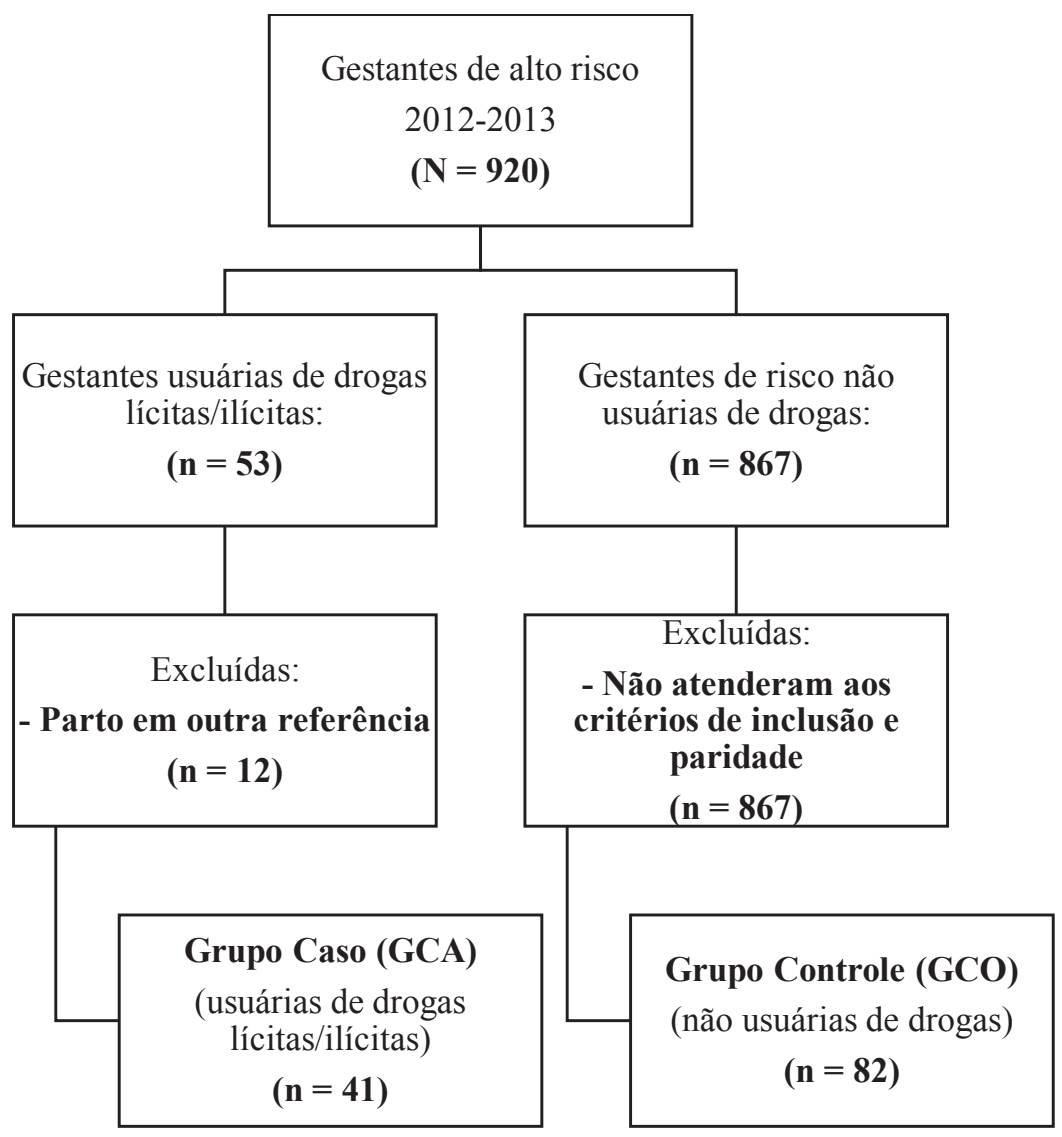

Figura 1 - Constituição da amostra

Tabela 1 - Características das gestantes de alto risco do Grupo Caso (usuárias de drogas lícitas/ilícitas) e do Grupo Controle (não usuárias de drogas) acompanhadas no ambulatório de risco. Maringá, PR, Brasil, 2014

\begin{tabular}{|c|c|c|c|c|}
\hline \multirow{2}{*}{$\begin{array}{c}\text { Grupo } \\
\text { Variáveis }\end{array}$} & \multicolumn{2}{|c|}{$\begin{array}{l}\text { Usuárias de Drogas } \\
\text { (lícitas/ilícitas) } \\
n=41\end{array}$} & \multicolumn{2}{|c|}{$\begin{array}{c}\text { Grupo Controle } \\
\text { (não usuárias) } \\
\text { n = } 82\end{array}$} \\
\hline & $\mathrm{n}$ & $\%$ & $\mathbf{n}$ & $\%$ \\
\hline \multicolumn{5}{|l|}{ Idade } \\
\hline 10 a 18 anos & 04 & 9,7 & 08 & 9,7 \\
\hline 19 a 35 anos & 28 & 68,3 & 56 & 68,3 \\
\hline 36 anos ou mais & 09 & 22,0 & 18 & 22,0 \\
\hline
\end{tabular}




\begin{tabular}{|c|c|c|c|c|}
\hline \multirow{2}{*}{$\begin{array}{c}\text { Grupo } \\
\text { Variáveis }\end{array}$} & \multicolumn{2}{|c|}{$\begin{array}{l}\text { Usuárias de Drogas } \\
\text { (lícitas/ilícitas) } \\
\qquad n=41\end{array}$} & \multicolumn{2}{|c|}{$\begin{array}{c}\text { Grupo Controle } \\
\text { (não usuárias) } \\
n=82\end{array}$} \\
\hline & $\mathrm{n}$ & $\%$ & $\mathrm{n}$ & $\%$ \\
\hline \multicolumn{5}{|l|}{ Raça/Cor } \\
\hline Branca & 24 & 58,5 & 56 & 68,3 \\
\hline Preta & 02 & 4,9 & 02 & 2,4 \\
\hline Parda & 15 & 36,6 & 24 & 29,3 \\
\hline \multicolumn{5}{|l|}{ Situação Conjugal } \\
\hline Vive com o companheiro & 16 & 39,0 & 51 & 62,2 \\
\hline Não vive com o companheiro & 25 & 61,0 & 31 & 37,8 \\
\hline \multicolumn{5}{|l|}{ Escolaridade } \\
\hline$<8$ anos & 23 & 56,1 & 47 & 57,3 \\
\hline$\geq 8$ anos & 18 & 43,9 & 35 & 42,7 \\
\hline \multicolumn{5}{|l|}{ Renda (Salário mínimo)* } \\
\hline Nenhum rendimento & 23 & 56,1 & 36 & 43,9 \\
\hline $1-2$ & 17 & 41,4 & 44 & 53,7 \\
\hline$>2$ & 01 & 2,5 & 02 & 2,4 \\
\hline \multicolumn{5}{|l|}{ Número de Consultas ${ }^{\dagger}$} \\
\hline $1-3$ & 23 & 56,0 & 39 & 47,6 \\
\hline $4-6$ & 09 & 22,0 & 33 & 40,2 \\
\hline$\geq 7$ & 09 & 22,0 & 10 & 12,2 \\
\hline \multicolumn{5}{|l|}{ Número de Gestações ${ }^{\ddagger}$} \\
\hline 1 & 12 & 29,3 & 30 & 36,6 \\
\hline $2-3$ & 14 & 34,1 & 40 & 48,8 \\
\hline $4-5$ & 15 & 36,6 & 12 & 14,6 \\
\hline
\end{tabular}

*Salário mínimo: R\$ 788,00; †Consultas de pré-natal especializada no Ambulatório de Risco; łlnclui a gestação atual

Tabela 2 - Análise dos desfechos perinatais dos grupos caso e controle avaliados no ambulatório de risco gestacional. Maringá, PR, Brasil, 2014

\begin{tabular}{|c|c|c|c|c|c|c|c|c|c|}
\hline \multirow{3}{*}{ Variáveis } & \multicolumn{4}{|c|}{ Grupos } & \multirow{3}{*}{$\mathbf{R}^{*}$} & & & \multirow{3}{*}{$\mathbf{X}^{2}$} & \multirow{3}{*}{$P$} \\
\hline & \multicolumn{2}{|c|}{$\begin{array}{l}\text { Usuárias de drogas } \\
\qquad(n=41)\end{array}$} & \multicolumn{2}{|c|}{$\begin{array}{c}\text { Controle } \\
(n=82)\end{array}$} & & \multicolumn{2}{|c|}{$\mathrm{IC}^{\dagger}\left(\mathrm{RR}^{\star}\right)$} & & \\
\hline & $n$ & $\%$ & $n$ & $\%$ & & & & & \\
\hline $\begin{array}{l}\text { Prematuridade } \\
\text { (<37 semanas) }\end{array}$ & 16 & 43,2 & 70 & 24,8 & 2,64 & 1,14 & 6,06 & 5,40 & $0,02^{\ddagger}$ \\
\hline $\begin{array}{l}\text { Baixo peso ao nascer } \\
(<2500 \mathrm{~g})\end{array}$ & 7 & 17,1 & 3 & 3,7 & 5,42 & 1,32 & 22,22 & 6,58 & 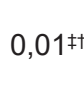 \\
\hline
\end{tabular}

A Tabela 2 continua na próxima página... 


\begin{tabular}{|c|c|c|c|c|c|c|c|c|c|}
\hline \multirow{3}{*}{ Variáveis } & \multicolumn{4}{|c|}{ Grupos } & \multirow{3}{*}{$\mathbf{R R}^{*}$} & & & \multirow{3}{*}{$X^{2}$} & \multirow{3}{*}{$\mathrm{P}$} \\
\hline & \multicolumn{2}{|c|}{$\begin{array}{l}\text { Usuárias de drogas } \\
\qquad(n=41)\end{array}$} & \multicolumn{2}{|c|}{$\begin{array}{l}\text { Controle } \\
(n=82)\end{array}$} & & \multicolumn{2}{|c|}{$\mathrm{IC}^{\dagger}\left(\mathrm{RR}^{*}\right)$} & & \\
\hline & $n$ & $\%$ & $\mathrm{n}$ & $\%$ & & & & & \\
\hline Apgar $1^{\circ}$ minuto $(<7)$ & 12 & 29,3 & 10 & 12,2 & 2,97 & 1,15 & 7,65 & 5,42 & $0,01^{\ddagger}$ \\
\hline Apgar $5^{\circ}$ minuto $(<7)$ & 4 & 9,8 & 3 & $3,7,1$ & 2,84 & 0,60 & 13,37 & 1,89 & 0,16 \\
\hline Parto normal & 9 & 22,0 & 27 & 32,9 & 0,57 & 0,23 & 1,17 & 1,36 & 0,20 \\
\hline Parto cesárea & 32 & 78,0 & 55 & 67,1 & 1,74 & 0,73 & 4,17 & 1,59 & 0,20 \\
\hline
\end{tabular}

${ }^{*}$ Risco relativo; †IC - Índice de Confiança; łp significativo considerando nível de significância de 5\%

\section{Discussão}

As repercussões decorrentes do uso de drogas lícitas/ilícitas na gestação têm se destacado em vários estudos, entretanto poucos apresentam os desfechos perinatais comparados com outros grupos vulneráveis na gestação, por serem escassos os serviços especializados na gestação de alto risco.

Neste estudo, foi possível comparar dois grupos (Caso e Controle) provenientes de um ambulatório especializado que oferece assistência focada na gestação de alto risco. Vale destacar que o serviço pertence à atenção secundária de saúde (ASS) e, mesmo com o atendimento direcionado, todas as gestantes devem continuar o pré-natal na atenção primária de saúde (APS), ou seja, na Unidade Básica de Saúde (UBS) de origem, a fim de não perder o vínculo com a equipe de sua área.

Mesmo com todo o acompanhamento da APP e ASS, o uso de drogas lícitas/ilícitas pelas gestantes causa menor adesão aos serviços e interrupção dos tratamentos propostos pela equipe de saúde. Esse comportamento está na maioria das vezes associado a problemas psicossociais ou a outros riscos gestacionais, acometendo-as em intercorrências obstétricas, gerando resultados desfavoráveis maternos e perinatais ${ }^{(3)}$.

Nesse sentido, a busca ativa de pacientes faltosas em consultas de pré-natal e o acompanhamento de equipes multidisciplinares são ações importantes para a reintegração da gestante usuária de drogas lícitas/ ilícitas nos tratamentos indicados, além disso, favorece à equipe de saúde diagnósticos precisos e assistência integral com perspectivas positivas aos desdobramentos maternos e perinatais.

Neste estudo, ao analisar os desfechos prematuridade e baixo peso ao nascer, os resultados apresentaram dados estatísticos significativos $(p=0,02)$ e $(p=0,01)$, respectivamente. Corroborando com esta pesquisa, uma meta-análise realizada com publicações de 1966 a 2009 apresentou 31 estudos, em que o uso de cocaína durante a gravidez foi associado ao parto prematuro odds ratio (OR), 3,38; intervalo de confiança (IC) 95\%, 2.72-4.21; baixo peso ao nascer (OR, 3,66; IC 95\%, 2,90-4,63); e crianças pequenas para a idade gestacional (OR, 3,23; IC 95\%, 2.43-4.30); menor idade gestacional no parto (-1,47 semana, IC 95\%, -1,97 para $-0,98$ semana); e peso ao nascer reduzido ( $-492 \mathrm{~g}$; IC $95 \%,-562$ a $-421 \mathrm{~g})^{(8)}$.

Além da cocaína, o uso de crack na gravidez também leva ao retardo do crescimento intrauterino, baixo peso ao nascer e aumenta o risco de parto prematuro ${ }^{(9-10)}$. Já em outro estudo, observou-se que a gestante usuária de crack pode ter complicações relacionadas ao descolamento prematuro da placenta, ruptura uterina, ruptura hepática, isquemia cerebral, infarto e morte. Outra droga ilícita comumente consumida por gestantes, com prevalência anual de uso entre 2,6 e 5,0\%, é a maconha e seu efeito ao recém-nascido (RN) foi coincidido com o mau desenvolvimento do tubo neural e possíveis anencefalias ${ }^{(6)}$.

Já quanto ao uso de cigarro, em um estudo de caso controle realizado no Piauí, com 168 puérperas, sendo 24 fumantes (caso) e 144 não fumantes (controle), não houve diferença estatística significativa para tempo de gestação $(38.63 \pm 0.31 ; 39.08 \pm 0.11)$ e peso ao nascer (3011 $\pm 1,98 ; 3110 \pm 0,55)$, entretanto as fumantes apresentaram menores índices de Apgar no $1^{\circ}$ e $5^{\circ}$ minutos (1': $7.04 \pm 0.19 ; 8.25 \pm 0.09$ e $5^{\prime}: 8.83 \pm 0.24$; $9.69 \pm 0.078, p<0,001)$ e maior número de parto cesárea $(54,17 \%)^{(11)}$.

Em discordância, pesquisa ${ }^{(12)}$ realizada no Japão, referente a efeitos adversos do uso do cigarro por mulher e homem sobre os resultados perinatais, em partos realizados entre 1997 e 2010, identificou efeitos da mãe fumante relacionados ao baixo peso ao nascer, feto pequeno para idade gestacional e circunferência cefálica diminuída, enquanto os efeitos do pai fumante foram relacionados a feto pequeno para idade gestacional e circunferência cefálica diminuída. No modelo ajustado, 
ambos os pais fumantes mostraram associações com baixo peso ao nascer (OR = 1,64, IC 95\% 1,18-2,27).

Além das drogas supracitadas, o consumo de álcool tem aumentado no público feminino e, embora poucos fossem os efeitos identificados nas primeiras horas de vida, existe uma grande preocupação nas consequências ao binômio. Em um estudo realizado em São Paulo, com 150 puérperas, sendo 20,7\% consideradas consumidoras de álcool pelo questionário T-ACE, identificou-se associação estatística significativa à restrição do crescimento fetal, sendo os fetos do sexo feminino aparentemente mais suscetíveis aos efeitos do álcool(13).

Nesse contexto, é possível inferir que o uso de drogas lícitas/ilícitas na gravidez representa um risco toxicológico ao binômio mãe/feto, uma vez que os estudos supracitados indicam desfechos desfavoráveis na gestação, além disso, a vinculação afetiva entre o binômio poderá ser prejudicada, já que o RN dependerá de cuidados da equipe de saúde. Vale ressaltar que o único contato externo do feto desde a fase embrionária é a mãe, sendo assim, após o nascimento, esse contato é vital, pois, além do vínculo afetivo, o uso de drogas pode prejudicar a amamentação por desequilíbrio comportamental apresentado pela mãe e/ou pelos efeitos colaterais que determinadas drogas podem causar ao RN.

\section{Conclusão}

Os resultados apresentados nesta pesquisa avigoram a literatura no que diz respeito aos desfechos desfavoráveis causados pelo uso de drogas lícitas/ ilícitas ao RN, reforçando os cuidados e orientações de enfermagem durante o pré-natal de risco habitual na atenção primária e/ou em ambulatório especializado na atenção secundária, assim é importante que o profissional conheça o perfil das gestantes de sua área ou do ambulatório que está realizando o pré-natal e, além disso, conheça os diversos tipos de drogas e seus efeitos, oferecendo suporte necessário à mulher, a fim de evitar, tratar e/ou eliminar este risco/vício em sua vida.

A limitação do presente estudo deve-se ao fato de envolver dados secundários, o que depende dos registros realizados pelos profissionais de saúde e do tamanho da amostra por se tratar de gestantes acompanhadas em um ambulatório específico ao prénatal de risco.

Os resultados encontrados nesta pesquisa mostram que gestantes que fazem uso de drogas lícitas/ilícitas estão suscetíveis a apresentarem como desfecho perinatal desfavorável a prematuridade, BPN e baixo valor Apgar no $1^{\circ}$ minuto, comprometendo a vitalidade do RN e da própria mãe. Sendo assim, sugerem-se novos estudos em relação às consequências neonatais e infantis decorrentes do uso de drogas lícitas/ilícitas na gestação, além do acompanhamento dessas crianças durante a amamentação, internações e desenvolvimento até um ano de vida.

\section{Referências}

1. World Health Organization (WHO). Global health risks: mortality and burden of disease attributable to selected major risks. Geneva: WHO; 2009. [cited 2016 May 6]. Available from: http://www.who.int/healthinfo/global_ burden_disease/GlobalHealthRisks_report_full.pdf

2. United Nations Office on Drugs and Crime (UNODC). World drug report 2015. New York, United Nations; 2015. [cited 2016 May 13]. Available from: <https://www.unodc. org/lpo-brazil/pt/frontpage/2015/06/relatorio-mundialsobre-drogas-de-2015--o-uso-de-drogas-e-estavel--maso-acesso-ao-tratamento-da-dependencia-e-do-hiv-aindae-baixo.html>

3. Kassada DS, Marcon SS, Pagliarini MA, Rossi RM. Prevalence of drug abuse among pregnant women. Acta Paul Enferm. 2013;26(5):467-71. doi: http://dx.doi. org/10.1590/S0103-21002013000500010

4. Ministério da Saúde (BR). Secretaria de Atenção à Saúde. Departamento de Ações Programáticas Estratégicas. Gestação de alto risco: manual técnico. 5 . ed. Brasília, DF; 2012. [Acesso 10 mai 2016]. Disponível em: http://bvsms.saude.gov.br/bvs/publicacoes/manual_ tecnico_gestacao_alto_risco.pdf

5. Maia JA, Pereira LA, Menezes FA. Consequências do uso de drogas durante a gravidez. Rev Enferm Contemporânea. 2015;4(2):121-8.

6. Botelha APM, Rocha RC, Melo VH. Uso e dependência de cocaína/crack na gestação, parto e puerpério. Rev Femina. 2013;41(1):23-32.

7. Portela GLC, Barros LM, Frota NM, Landim APP, Caetano JA, Farias FLR. Percepção da gestante sobre o consumo de drogas ilícitas na gestação. SMAD, Rev. Eletrônica Saúde Mental Álcool Drog. 2013;9(2):58-63.

8. Gouin K, Murphy K, Prakesh SS. Effect of cocaine use during pregnancy on low birth weight and preterm birth: systematic review and metanalyses. Am J Obstet Gynecol. 2011; 204(4):340.e1-340.e12.

9. Marques ACPR, Ribeiro M, Laranjeira RR, Andrada NC. Abuso e dependência: crack. Rev Assoc Méd Bras. 2012;58(2):141-53.

10. Alencar JCG, Junior CAA, Matos AMB. "Crack Babies": uma revisão sistemática dos efeitos em recém-nascidos 
e em crianças do uso do crack durante a gestação. Rev Pediatria SOPERJ. 2011;12(1):16-21.

11. Sousa JRPC, Macedo LC, Neto JDVN. Os efeitos do tabagismo sobre a incidência de partos pré-termos. Rev Interdisciplinar. 2011;4(3):9-13.

12. Inoue $S$, Naruse $H$, Yorifuji T, Kato T, Murakoshi T, Doi $\mathrm{H}$, et al. Impacto of maternal and paternal smoking on birth outcomes. J Public Health. (Oxf). 2016; 1-10.

13. Freire TM, Machado JC, Melo EV, Melo DG. Efeitos do consumo de bebida alcoólica sobre o feto. Rev Bras Ginecol Obstet. 2005;27(7):376-81. doi: https://doi.org/10.11606/ issn.1806-6976.v9i2p58-63 